\title{
On the Mechanism of Shear-Thinning in Viscous Oppositely Charged Polyelectrolyte Surfactant Complexes (PESCs)
}

\author{
Giuseppe Rosario Del Sorbo, Sylvain Prévost, Emanuel Schneck, Michael Gradzielski,* \\ and Ingo Hoffmann*
}

Cite This: J. Phys. Chem. B 2020, 124, 909-913

Read Online

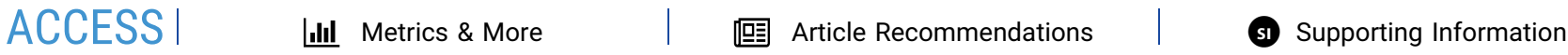

ABSTRACT: Semidilute mixtures of the cationically modified cellulose-based polyelectrolyte JR 400 and the anionic surfactant sodium dodecyl sulfate (SDS) form highly viscous solutions if a slight excess of charges from the polyelectrolyte is present. The reason for this is the formation of mixed rodlike aggregates in which the surfactant cross-links several polyelectrolyte chains. The same solutions also show shear-thinning behavior. In this paper, we use rheoSANS to investigate the structural evolution of the rodlike aggregates under steady shear and thereby elucidate the

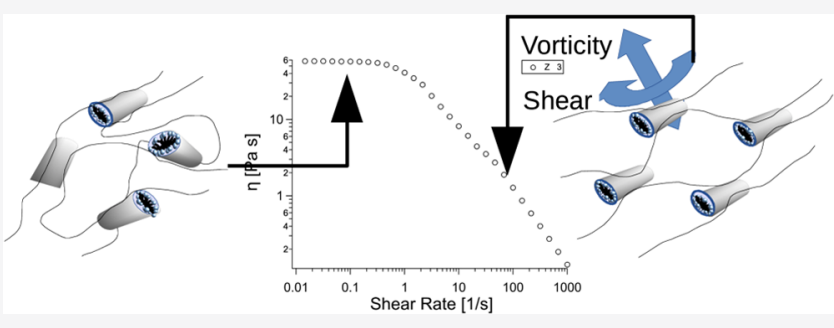
mechanism of shear-thinning in these viscous oppositely charged polyelectrolyte surfactant complexes.

\section{INTRODUCTION}

Oppositely charged polyelectrolyte (PE) surfactant complexes (PESCs) show an extremely rich self-aggregation behavior. ${ }^{1-4}$ While such systems have attracted the interest of researchers for a few decades now, ${ }^{5}$ their behavior is still not fully understood. Some recent reviews ${ }^{6-8}$ give an overview of our current understanding of PESCs. Some of these systems do not only show structural organization on the mesoscale but also influence the macroscopically observed viscosity similar to systems with hydrophobically modified PEs. ${ }^{9-11}$ The addition of relatively small amounts of surfactant can be sufficient to have a massive impact on the solution viscosity relative to the pure polyelectrolyte solution. Depending on the system and the exact composition, a decrease ${ }^{12-14}$ or an increase $e^{15-20}$ of viscosity can be observed. It was found that in mixtures of the cationically modified hydroxyethycellulose JR 400 and different anionic surfactants, an increase in viscosity is related to the formation of mixed aggregates, ${ }^{18,21}$ while in mixtures of carboxymethyl cellulose (CMC) and different alkyltrimethylammonium bromides, a decrease in viscosity due to the shrinkage of the PE chain was observed, ${ }^{13}$ and the viscosity of solutions with sodium hyaluronate is hardly affected by the addition of a cationic surfactant ${ }^{14}$ even though small-angle neutron scattering (SANS) shows the formation of large aggregates.

RheoSANS allows to monitor structural changes using SANS while samples are under shear. This method has been used to great success for wormlike micelles and other colloidal systems. $^{22-29}$ Here, we used it to elucidate the mechanism behind the shear-thinning behavior in oppositely charged PESCs.
Aqueous mixtures of the cationically modified hydroxyethyl cellulose JR 400 and SDS show a remarkable increase in viscosity by several orders of magnitude relative to the pure $\mathrm{PE}$ solution at the same PE concentration. ${ }^{5}$ Near charge equilibrium, a 2-phase region consisting of a dilute phase and a precipitated PE-surfactant complex phase is present, while both with a surfactant or a PE excess, a clear homogeneous phase is formed. The increase in viscosity is observed in the PE excess phase near the phase boundary to the 2-phase region above the overlap concentration, i.e., in the semidilute concentration range. This is the case for the solutions with 1 wt $\% \mathrm{JR} 400^{30,31}$ (corresponding to $10 \mathrm{mM}$ of PE charges) studied here. A graph of the zero shear viscosity $\eta_{0}$ as a function of the charge ratio $Z$, defined as $Z=[\mathrm{PE}$ charges $] /[$ surfactant charges $]$ can be found in the Supporting Information Figure $S 1$. A noticeable increase of the viscosity is observed for charge ratios of about $Z=10$ (corresponding to 1 $\mathrm{mM}$ surfactant for a $1 \mathrm{wt} \% \mathrm{JR} 400$ solution) and less up to the onset of the 2 -phase region around $Z=2.5$. Combining smallangle neutron scattering (SANS) and neutron spin-echo (NSE) spectroscopy, it was shown that this increase in viscosity is due to the formation of rodlike aggregates with a diameter similar to that of a surfactant micelle and a length of about $50 \mathrm{~nm}^{32}$ This is different from wormlike micellar systems or stiff, strongly anisotropic colloids ${ }^{33,34}$ in that no overlap between the aggregates themselves is needed to obtain

Received: November 7, 2019

Revised: January 13, 2020

Published: January 14, 2020 
viscous samples, as they only act as cross-links between PE chains and therefore relatively small surfactant concentrations of $3 \mathrm{mM}$ and less are sufficient to obtain viscous samples, while surfactant concentrations in viscous wormlike micellar solutions are typically on the order of some $10 \mathrm{mM}^{35,36}$ These aggregates consist of both surfactant and PE and act as cross-links for the PE as they contain several PE chains. While the PE does not form a dense layer around the surfactant core of the aggregates, careful analysis of the data from different scattering contrasts shows that it is nevertheless found predominantly on the outside of the aggregates. ${ }^{21}$ For this increase in viscosity to take place, it is important that the $\mathrm{PE}$ concentration is sufficiently high. While $1 \mathrm{wt} \%$ of JR 400 is enough to observe a strong effect, reducing its concentration to 0.3 wt \%, hardly any effect can be observed. Using SANS, it has been shown that this is not because of the absence of the rodlike aggregates but because of a subtle change in their composition. ${ }^{21}$ Decreasing the PE concentration leads to a decrease in the number of PE chains found in a single aggregate, and as the number of $\mathrm{PE}$ chains per aggregate approaches 1, they lose their ability to act as cross-links between chains. Another important finding is the fact that the $\mathrm{PE}$ chains are not frozen in the aggregates but retain a rather high degree of freedom of movement even when incorporated in them.

The same solutions display shear-thinning behavior beyond a critical shear rate $\dot{\gamma}_{\text {crit }}$ (see Figure S2) and here we use rheoSANS to investigate the underlying mechanism leading to this behavior. This is not only an interesting question from fundamental science but also highly relevant for applications, as such mixtures are frequently employed in formulations from detergency, cosmetics, shampoos, etc., where they are applied under shearing conditions. In principle, two different scenarios are thinkable. (I) Under shear, PE chains are torn out of the aggregates, reducing their number per aggregate and consequently reducing the aggregate's efficiency as crosslinker, which ultimately leads to a situation comparable to what is observed at low PE concentrations. In this scenario, the radially averaged scattering intensity would decrease noticeably in the Q-range where the aggregates are observed (see the Supporting Information Figures S3 and S4). (II) Knowing from NSE that the PE chains are not frozen in the aggregates and assuming that they can move mostly along the long axis of the aggregates, it would be sufficient if the aggregates align along the direction of shear. In this scenario, the radially averaged intensity of the radial rheoSANS data (1-3 plane) would remain identical, but the scattering pattern would become anisotropic.

\section{MATERIALS AND METHODS}

Small-angle neutron scattering (SANS) measurements were performed on the instrument D11 at the Institut LaueLangevin (ILL, Grenoble, France) using a neutron wavelength of $5.0 \AA$ and sample-to-detector distances of $1.4,8$, and $39 \mathrm{~m}$, covering a $Q$-range from 0.022 to $4 \mathrm{~nm}^{-1}$. The rheoSANS setup consists of an Anton-Paar Physica MCR 501 rheometer with a titanium Couette geometry of $30 \mathrm{~mm}$ diameter and 0.5 mm gap. SANS data were reduced using the software package BerSANS, ${ }^{37}$ using the direct beam flux to obtain data on absolute scale.

The anisotropy analysis of the data was performed using the software SASET. ${ }^{38}$ Principal component analysis (PCA) was used to quantify anisotropy and determine the maximum scattering direction, by finding the vector onto which the projected data, weighed by their intensity, have a maximum variability. For the anisotropy analysis using PCA, only Qvalues covering $2 \pi$ azimuthally of the $8 \mathrm{~m}$ measurements were used. The resulting $Q$-range is $0.125-0.725 \mathrm{~nm}^{-1}$. Additionally, $45^{\circ}$ sector averages parallel $\left(0^{\circ}\right)$ and perpendicular $\left(90^{\circ}\right)$ to the direction of shear were taken.

The radially averaged intensity of rodlike particles can be modeled as a linear combination of the intensity from the aggregates and the intensity from free PE chains. The aggregates consist of all of the surfactant in the sample and a fraction of the PE $x_{\mathrm{pa}}$, so that their volume fraction reads

$$
\phi_{\mathrm{agg}}=\phi_{\mathrm{surf}}+\phi_{\mathrm{PE}} x_{\mathrm{pa}}
$$

where $\phi_{\text {surf }}$ and $\phi_{\mathrm{PE}}$ are the volume fractions of the surfactant and $\mathrm{PE}$, respectively. Both the free $\mathrm{PE}$ and the aggregates were modeled as cylinders.

Assuming a homogeneous structure of the aggregates along their long axis, we can calculate the average number of PE chains per aggregate $N_{\mathrm{c}}$ by relating the volume fractions of surfactant and $\mathrm{PE}$ in the aggregates to the radii of the whole aggregate and individual $\mathrm{PE}$ chains

$$
N_{\mathrm{c}}=\frac{x_{\mathrm{pa}} \phi_{\mathrm{PE}}}{x_{\mathrm{pa}} \phi_{\mathrm{PE}}+\phi_{\text {surf }}} \frac{R_{\mathrm{agg}}^{2}}{R_{\mathrm{PE}}^{2}}
$$

where $R_{\mathrm{agg}}$ is the radius of the mixed aggregates and $R_{\mathrm{PE}}$ is the radius of an individual PE chain. At $N_{\mathrm{c}}<2$, no pronounced increase in viscosity can be observed as the aggregates do not act as efficient cross-links anymore. See the Supporting Information for more details.

Complementary offline rheology measurements were performed using an Anton-Paar MCR 501 rheometer in cone/plate geometry with a $50 \mathrm{~mm}$ cone diameter and $1^{\circ}$ cone angle. All measurements were carried out at $25{ }^{\circ} \mathrm{C}$.

Sodium dodecyl sulfate (SDS, $98.5 \%$ ) was purchased from Sigma-Aldrich and used without further purification.

The cationically modified hydroxyethyl cellulose JR 400 (Dow Chemical) has a molecular weight of about $500 \mathrm{~kg} / \mathrm{mol}$ (PDI $=1.85,{ }^{39} 27 \%$ of the glucose units are cationically modified, resulting in $1000 \mathrm{~g}$ of $\mathrm{PE}$ per mol of positive charges). ${ }^{10}$ See the Supporting Information Figure S8 for its chemical structure. Its overlap concentration was found to be between $0.8^{31}$ and $0.25 \mathrm{wt} \%{ }^{30}$ so that samples with a $\mathrm{PE}$ concentration of $1 \mathrm{wt} \%$ are well in the semidilute regime.

The concentration of surfactant is expressed as the charge ratio $Z$, which is given by $Z=[\mathrm{PE}$ charges $] /[$ surfactant charges]. All samples had a JR 400 concentration of 1 wt $\%$ corresponding to $10 \mathrm{mM}$ of charges. All solutions were prepared in $\mathrm{D}_{2} \mathrm{O}$ (Euriso-top, France).

\section{RESULTS AND DISCUSSION}

Performing rheoSANS measurements on viscous, shearthinning PESCs allows to differentiate between these two scenarios. While a reduction of the cross-linking efficiency of the rodlike aggregates by the reduction of the number of PE chains in the aggregates would reduce the scattering intensity, an alignment of the aggregates would only result in an anisotropic scattering pattern, leaving the radially averaged intensity unchanged.

As can be seen in Figures 1 and S5, the radially averaged intensity is independent of the applied shear rate at shear rates significantly above the critical shear rate $\left(0.9 \mathrm{~s}^{-1}\right.$ for $\left.Z=3\right)$, 


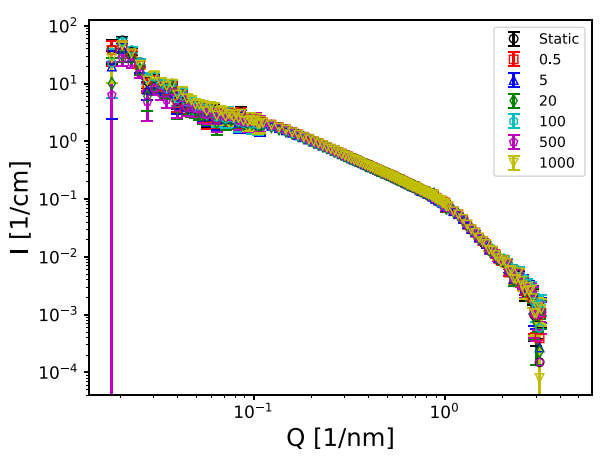

Figure 1. SANS curves for the sample with $Z=3$ at different shear rates indicated in the graph. The SANS intensity remains unchanged, while a reduction of the number of chains per aggregate should result in a small but noticeable difference as shown in Figures S3 and S4.

even though a small, yet noticeable, difference should be observed if the number of chains per aggregate would be reduced. Specifically, the intensity should be reduced in the intermediate $Q$ range and somewhat increased in the high $Q$ range because of the decrease of the volume fraction of mixed aggregates and an increase of the volume fraction of free PE chains (see Figures S3 and S4 for the theoretical curves in which we varied the fraction of JR 400 in the mixed aggregates $x_{\mathrm{pa}}$ and Figure S5 for a magnification of the mid- $Q$ range of Figure 1). This allows us to exclude the first scenario, where shear-thinning is caused by the disruption of the cross-linking mixed aggregates.

At first glance, no anisotropy is visible in the twodimensional (2D) detector images (see Figure 2). Taking
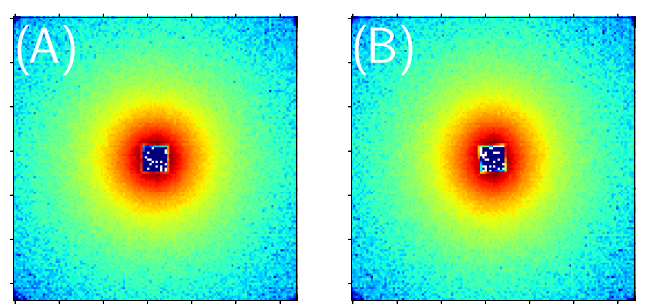

Figure 2. Two-dimensional (2D) detector images for sample with $Z=$ 3. (A) At rest and (B) at $\dot{\gamma}=500 \mathrm{~s}^{-1}$. No pronounced anisotropy can be seen.

$45^{\circ}$ sector averages on the other hand with different angles relative to the direction of shear shows no difference at rest (see Figure S6) and a small but significant difference under shear (see Figure S7), which shows that we induce anisotropy by shearing the sample. These anisotropic scattering patterns were now analyzed in a quantitative fashion using principal component analysis (PCA). ${ }^{38,40}$ PCA is an established method from pattern recognition that consists in finding a succession of orthogonal vectors accounting for the highest variability in a data set. Applied to 2D intensity data, the main component indicates the direction and breadth of maximum dispersion in the intensities, i.e., the main axis of anisotropy and its amplitude. It yields an anisotropy parameter $A$ with $A=0$ for a completely isotropic scattering pattern and $A=1$ for scattering only along one direction. Performing PCA on the data at mid$Q$ where the rodlike aggregates are seen shows that weak anisotropy forms as the shear rate is increased. The effects are negligible for pure JR 400 and the sample with $Z=10$, which have a rather low viscosity. It becomes far more pronounced for the more viscous samples with $Z=5$ and 3 (see Figure 3), It can also be seen that the onset of the increase in anisotropy

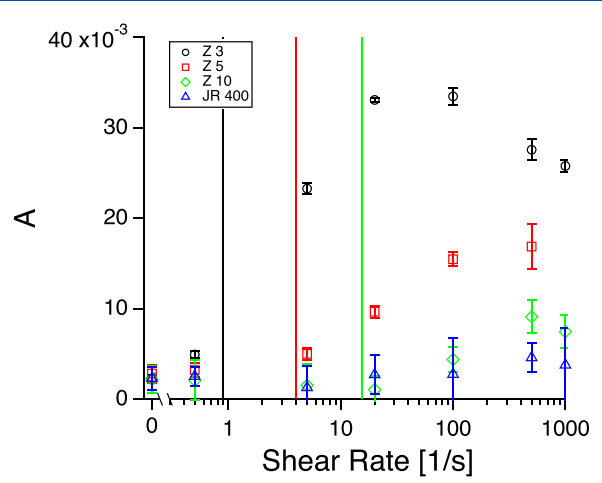

Figure 3. Anisotropy parameter $A$ from PCA for samples indicated in the graph using the mid- $Q$ configuration $(8 \mathrm{~m}$ sample-to-detector distance). The more viscous samples with $Z=3$ and 5 show an increase of anisotropy with the shear rate. The critical shear rates $\dot{\gamma}_{\text {crit }}$ of the samples as determined from viscometry (see Figure S2) are indicated in the graph as vertical lines. The onset of the increase of $A$ roughly corresponds to $\dot{\gamma}_{\text {crit }}$.

is at significantly lower shear rates for $Z=3$ than for $Z=5$, which is in agreement with the significantly lower $\dot{\gamma}_{\text {crit }}$ at $Z=3$ $\left(0.9\right.$ vs $\left.4 \mathrm{~s}^{-1}\right)$. The onset of anisotropy and the critical shear rate are in good agreement, which indicates that this structural change is at the origin of the macroscopic change of the flow behavior.

\section{CONCLUSIONS}

In summary, using rheoSANS, we were able to rule out that shear-thinning in our oppositely charged polyelectrolyte

\section{At Rest}

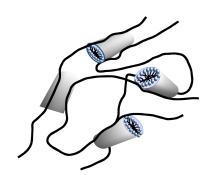

\section{Applied Shear}

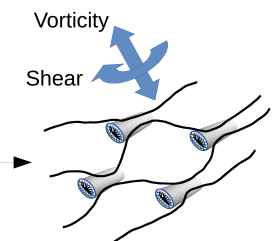

Figure 4. RheoSANS measurements support scenario (II): Applying shear, the fraction of free PE chains does not increase. The reason for the shear-thinning behavior of the solutions is an alignment of the rodlike aggregates in the direction of flow, which allows the PE chains to move along the flow direction more easily.

surfactant system is related to the rupture of the mixed rodlike aggregates that were found to be responsible for the increase in viscosity previously. In fact, it is observed that a slight orientation of the rodlike aggregates is sufficient. This matches our previous observation from NSE, where it was found that the PE retains some of its freedom of movement even in the aggregates. This motion might be mostly along the long axis of the aggregates, and orienting them in the direction of shear allows for easier relaxation of shear stress and this orientation of aggregates makes the system shear-thinning. An interesting detail can be seen when inspecting Figure 3 more carefully. For $Z=3$ at the two highest shear rates, a slight decrease in the anisotropy can be seen. This might indicate that beyond a certain shear rate, the decrease of viscosity is governed by an 
increase of mobility of the PE chains in the aggregates. In principle, rheoNSE measurements ${ }^{41}$ might be able to clarify this point in the future (Figure 4).

\section{ASSOCIATED CONTENT}

\section{(3) Supporting Information}

The Supporting Information is available free of charge at https://pubs.acs.org/doi/10.1021/acs.jpcb.9b10467.

Additional measured and simulated SANS curves, details on the theory for the simulated curves and additional rheology data (PDF)

\section{AUTHOR INFORMATION}

\section{Corresponding Authors}

Michael Gradzielski - Stranski-Laboratorium für Physikalische und Theoretische Chemie, Institut für Chemie, Technische Universität Berlin, D-10623 Berlin, Germany; ๑ orcid.org/ 0000-0002-7262-7115; Email: michael.gradzielski@tuberlin.de

Ingo Hoffmann - Institut Max von Laue-Paul Langevin (ILL), F-38042 Grenoble, France; ๑ orcid.org/0000-0001-71786467; Email: hoffmann@ill.fr

\section{Authors}

Giuseppe Rosario Del Sorbo - Max Planck Institute of Colloids and Interfaces, 14476 Potsdam, Germany; Institut Max von Laue-Paul Langevin (ILL), F-38042 Grenoble, France

Sylvain Prévost - Institut Max von Laue-Paul Langevin (ILL), F-38042 Grenoble, France; ๑ orcid.org/0000-0002-60081987

Emanuel Schneck - Max Planck Institute of Colloids and Interfaces, 14476 Potsdam, Germany; Soft Matter Biophysics, Department of Physics, TU Darmstadt, D-64289 Darmstadt, Germany; $\odot$ orcid.org/0000-0001-9769-2194

Complete contact information is available at:

https://pubs.acs.org/10.1021/acs.jpcb.9b10467

\section{Notes}

The authors declare no competing financial interest.

\section{ACKNOWLEDGMENTS}

Allocation of beamtime by the ILL is gratefully acknowledged. G.R.D.S. thanks the ILL for a doctoral scholarship through the ILL $\mathrm{PhD}$ program. E.S. gratefully acknowledges financial support by the Max Planck Society and by the German Research Foundation (DFG) via Emmy-Noether grant (SCHN 1396/1). The authors would like to thank Dr. Lionel Porcar (ILL) for his help setting up the rheometer at D11. The raw data of the rheoSANS experiment is accessible under $\mathrm{dx}$. doi.org/10.5291/ILL-DATA.9-11-1798.

\section{REFERENCES}

(1) Goddard, E. Polymer-surfactant interaction part II. Polymer and surfactant of opposite charge. Colloids Surf. 1986, 19, 301-329.

(2) Holmberg, K.; Jönsson, B.; Kronberg, B.; Lindman, B. Surfactants and Polymers in Aqueous Solution; John Wiley \& Sons, Ltd.: Chichester, UK, 2002.

(3) Chiappisi, L.; Hoffmann, I.; Gradzielski, M. Complexes of oppositely charged polyelectrolytes and surfactants - recent developments in the field of biologically derived polyelectrolytes. Soft Matter 2013, 9, 3896-3909.
(4) Chiappisi, L.; Prevost, S.; Grillo, I.; Gradzielski, M. Chitosan/ alkylethoxy carboxylates: A surprising variety of structures. Langmuir 2014, 30, 1778-1787.

(5) Goddard, E. D.; Hannan, R. B. Cationic polymer/anionic surfactant interactions. J. Colloid Interface Sci. 1976, 55, 73-79.

(6) Ferreira, G. A.; Loh, W. Liquid crystalline nanoparticles formed by oppositely charged surfactant-polyelectrolyte complexes. Curr. Opin. Colloid Interface Sci. 2017, 32, 11-22.

(7) Khan, N.; Brettmann, B. Intermolecular interactions in polyelectrolyte and surfactant complexes in solution. Polymers 2019, 11, No. 51.

(8) Gradzielski, M.; Hoffmann, I. Polyelectrolyte-surfactant complexes (PESCs) composed of oppositely charged components. Curr. Opin. Colloid Interface Sci. 2018, 35, 124-141.

(9) Wang, K. T.; Iliopoulos, I.; Audebert, R. Viscometric behaviour of hydrophobically modified poly(sodium acrylate). Polym. Bull. 1988, 20, 577-582.

(10) Thuresson, K.; Nilsson, S.; Lindman, B. Effect of hydrophobic modification on phase behavior and rheology in Mixtures of Oppositely Charged Polyelectrolytes. Langmuir 1996, 12, 530-537.

(11) Nyström, B.; Kjøniksen, A.-L.; Beheshti, N.; Zhu, K.; Knudsen, K. D. Rheological and structural aspects on association of hydrophobically modified polysaccharides. Soft Matter 2009, 5, 1328-1339.

(12) Guillot, S.; McLoughlin, D.; Jain, N.; Delsanti, M.; Langevin, D. Polyelectrolyte-surfactant complexes at interfaces and in bulk. J. Phys.: Condens. Matter 2003, 15, S219-S224.

(13) Jain, N.; Trabelsi, S.; Guillot, S.; McLoughlin, D.; Langevin, D.; Letellier, P.; Turmine, M. Critical Aggregation Concentration in Mixed Solutions of Anionic Polyelectrolytes and Cationic Surfactants. Langmuir 2004, 20, 8496-8503.

(14) Buchold, P.; Schweins, R.; Di, Z.; Gradzielski, M. Structural behaviour of sodium hyaluronate in concentrated oppositely charged surfactant solutions. Soft Matter 2017, 13, 2253-2263.

(15) Kästner, U.; Hoffmann, H.; Donges, R.; Ehrler, R. Interactions between modified hydroxyethyl cellulose (HEC) and surfactants. Colloids Surf., A 1996, 112, 209-225.

(16) Tsianou, M.; Alexandridis, P. Control of the rheological properties in solutions of a polyelectrolyte and an oppositely charged surfactant by the addition of cyclodextrins. Langmuir 1999, 15, 81058112.

(17) Beheshti, N.; Zhu, K.; Kjøniksen, A.; Nyström, B. Characterization of complexation and phase behavior of mixed systems of unmodified and hydrophobically modified oppositely charged polyelectrolytes. Colloid Polym. Sci. 2010, 288, 1121-1130.

(18) Hoffmann, I.; Heunemann, P.; Prévost, S.; Schweins, R.; Wagner, N. J.; Gradzielski, M. Self-aggregation of mixtures of oppositely charged polyelectrolytes and surfactants studied by rheology, dynamic light scattering and small-angle neutron scattering. Langmuir 2011, 27, 4386-4396.

(19) Alves, L.; Lindman, B.; Klotz, B.; Böttcher, A.; Haake, H.; Antunes, F. E. Rheology of polyacrylate systems depends strongly on architecture. Colloid Polym. Sci. 2015, 293, 3285-3293.

(20) Zhang, J.; Yu, W.; Zhang, R.; Yang, H. Rheological Study of Interactions between Anionic Guar and Oppositely Charged Surfactant. J. Dispersion Sci. Technology 2015, 36, 1290-1296.

(21) Hoffmann, I.; Farago, B.; Schweins, R.; Falus, P.; Sharp, M.; Prévost, S.; Gradzielski, M. On the mesoscopic origins of high viscosities in some polyelectrolyte-surfactant mixtures. J. Chem. Phys. 2015, 143, No. 074902.

(22) Stieger, M.; Richtering, W. Shear-induced phase separation in aqueous polymer solutions: Temperature-sensitive microgels and linear polymer chains. Macromolecules 2003, 36, 8811-8818.

(23) Förster, S.; Konrad, M.; Lindner, P. Shear thinning and orientational ordering of wormlike micelles. Phys. Rev. Lett. 2005, 94, No. 017803.

(24) Dreiss, C. A. Wormlike micelles: Where do we stand? Recent developments, linear rheology and scattering techniques. Soft Matter 2007, 3, 956-970. 
(25) Reinicke, S.; Karg, M.; Lapp, A.; Heymann, L.; Hellweg, T.; Schmalz, H. Flow-induced ordering in cubic gels formed by P2VP-bPEO-b-P(GME-co-EGE) triblock terpolymer micelles: A rheo-SANS Study. Macromolecules 2010, 43, 10045-10054.

(26) Rogers, S.; Kohlbrecher, J.; Lettinga, M. P. The molecular origin of stress generation in worm-like micelles, using a rheo-SANS LAOS approach. Soft Matter 2012, 8, 7831-7839.

(27) Eberle, A. P.; Porcar, L. Flow-SANS and rheo-SANS applied to soft matter. Curr. Opin. Colloid Interface Sci. 2012, 17, 33-43.

(28) Kundu, S. K.; Gupta, S.; Stellbrink, J.; Willner, L.; Richter, D. Relating structure and flow of soft colloids. Eur. Phys. J.: Spec. Top. 2013, 222, 2757-2772.

(29) Calabrese, M. A.; Rogers, S. A.; Murphy, R. P.; Wagner, N. J. The rheology and microstructure of branched micelles under shear. $J$. Rheol. 2015, 59, 1299-1328.

(30) Donnelly, M. W.; Hailemichael, M.; Liberatore, M. W. Altering the viscosity of cationically modified cellulose polymers by the addition of salt. J. Appl. Polym. Sci. 2015, 132, No. 41616.

(31) Hoffmann, I.; Prévost, S.; Medebach, M.; Rogers, S. E.; Wagner, N. J.; Gradzielski, M. Control of rheological behaviour with oppositely charged polyelectrolyte surfactant mixtures. Tenside, Surfactants, Deterg. 2011, 48, 488-494.

(32) Hoffmann, I.; Farago, B.; Schweins, R.; Falus, P.; Sharp, M.; Gradzielski, M. Structure and dynamics of polyelectrolytes in viscous polyelectrolyte-surfactant complexes at the mesoscale. Europhys. Lett. 2013, 104, No. 28001.

(33) Lang, C.; Kohlbrecher, J.; Porcar, L.; Lettinga, M. P. The connection between biaxial orientation and shear thinning for quasiideal rods. Polymers 2016, 8, No. 291.

(34) Lang, C.; Porcar, L.; Kriegs, H.; Lettinga, M. P. A quest for shear banding in ideal and non ideal colloidal rods. J. Phys. D: Appl. Phys. 2019, 52, No. 074003.

(35) Thareja, P.; Hoffmann, I. H.; Liberatore, M. W.; Helgeson, M. E.; Hu, Y. T.; Gradzielski, M.; Wagner, N. J. Shear-induced phase separation (SIPS) with shear banding in solutions of cationic surfactant and salt. J. Rheol. 2011, 55, 1375-1397.

(36) Takeda, M.; Kusano, T.; Matsunaga, T.; Endo, H.; Shibayama, M.; Shikata, T. Rheo-SANS studies on shear-thickening/thinning in aqueous rodlike micellar solutions. Langmuir 2011, 27, 1731-1738.

(37) Keiderling, U. The new 'BerSANS-PC' software for reduction and treatment of small angle neutron scattering data. Appl. Phys. A: Mater. Sci. Process. 2002, 74, S1455-S1457.

(38) Muthig, M.; Prévost, S.; Orglmeister, R.; Gradzielski, M. SASET: A program for series analysis of small-angle scattering data. J. Appl. Crystallogr. 2013, 46, 1187-1195.

(39) Li, D.; Kelkar, M. S.; Wagner, N. J. Phase behavior and molecular thermodynamics of coacervation in oppositely charged polyelectrolyte/surfactant systems: A cationic polymer JR 400 and anionic surfactant SDS mixture. Langmuir 2012, 28, 10348-10362.

(40) Bishop, C. M. Pattern Recognition and Machine Learning; Springer, New York, 2006.

(41) Kawecki, M.; Adlmann, F.; Gutfreund, P.; Falus, P.; Uhrig, D.; Gupta, S.; Farago, B.; Zolnierczuk, P.; Cochran, M.; Wolff, M. Direct measurement of topological interactions in polymers under shear using neutron spin echo spectroscopy. Sci. Rep. 2019, 9, No. 2823. 